\title{
NEW OPTIMIZED SPLINE FUNCTIONS FOR INTERPOLATION ON THE HEXAGONAL LATTICE
}

\section{Laurent Condat*}

\author{
Institute of Biomathematics and Biometry \\ Helmholtz Zentrum München, \\ German Res. Center for Environmental Health \\ Neuherberg, Germany
}

\author{
Dimitri Van De Ville \\ Biomedical Imaging Group (BIG) \\ École Polytechnique Fédérale de Lausanne \\ Lausanne, Switzerland
}

\begin{abstract}
We propose new discrete-to-continuous interpolation models for hexagonally sampled data, that generalize two families of splines developed in the literature for the hexagonal lattice, to say the hexsplines and three directional box-splines. This extension is inspired by the construction of MOMS functions in 1-D, that generalize and outperform classical 1-D B-splines [1]. Our new generators have optimal approximation theoretic performances, for exactly the same computation cost as their spline counterparts.
\end{abstract}

Index Terms - hexagonal sampling, three-directional mesh, interpolation, 2-D lattices, multi-dimensional splines, linear shift invariant signal spaces, approximation theory.

\section{INTRODUCTION}

Image interpolation consists in defining a spatially continuous function fitting a discrete image. This reconstruction process is essential to many analysis and resampling tasks such as image scaling, rotation, registration, edge detection... Most images are available on the Cartesian lattice $\mathbb{Z}^{2}$, although hexagonal sampling is known to have better geometrical and topological properties, and to enable a more efficient representation of 2-D signals [2,3]. Recent progress has brought hexagonal sampling into the domain of consumer electronics and there exists now imaging sensors that acquire on the hexagonal lattice, e.g. [4]. This is likely to foster a renewed interest for hexagonal image processing and justifies the study of methods specifically dedicated to the treatment of hexagonally sampled data.

The generic problem of interpolating data sampled on the lattice $\Lambda=\mathbf{R} \mathbb{Z}^{2}$ consists in estimating the function $f(\mathbf{x})$ from which we are only given the samples values $s[\mathbf{k}]=f(\mathbf{R k}), \mathbf{k} \in \mathbb{Z}^{2}$ at the lattice sites. In this work, we consider the hexagonal lattice, corresponding to

$$
\mathbf{R}=\sqrt{\frac{2}{\sqrt{3}}}\left[\begin{array}{cc}
1 & 1 / 2 \\
0 & \sqrt{3} / 2
\end{array}\right] .
$$

When $f$ is assumed to be band-limited, Shannon's theorem guarantees perfect reconstruction of $f(\mathbf{x})$ using interpolation with a lowpass cardinal-sine function. However, this ideal interpolator has an infinite support and its use generates visually disturbing ringing artifacts along edges. Instead, practitioners rely on a more localized generator $\varphi(\mathbf{x})$ with compact support. For images sampled on the

\footnotetext{
${ }^{*}$ The first author is supported by the Marie Curie Excellence Team Grant MEXT-CT-2004-013477, Acronym MAMEBIA, funded by the European Commission. Contact: laurent. condat@helmholtz-muenchen.de.
}

square lattice, separable extensions of 1-D algorithms are available, with a vast literature that mainly relies on piecewise polynomial functions. Spline models are particularly popular, since they conjugate optimal approximation properties with simplicity and easy implementation $[5,6]$. For data available on uniform hexagonal lattices, there are mainly two families of non-separable 2-D splines that exploit the geometric advantages of this sampling scheme, like its twelve-fold symmetry, to say, the hex-splines and the threedirectional box-splines. We present them briefly in Section 2 along with some basics of multi-dimensional interpolation. In Section 3, we propose new 2-D functions, which generalize these two families of splines. Our construction is inspired by the design of the family of MOMS functions in 1-D [1], that encompasses the B-splines as a particular case. In these extended set of functions, we choose new generators with better approximation theoretic performances as boxsplines and hex-splines for the same support size and degree, hence, an identical computation cost.

\section{SPLINE INTERPOLATION ON THE HEXAGONAL LATTICE}

We first introduce some notations. The Fourier transform of $f(\mathbf{x}) \in$ $L_{2}\left(\mathbb{R}^{2}\right)$ is $\hat{f}(\boldsymbol{\omega})=\int_{\mathbb{R}^{2}} f(\mathbf{x}) \exp \left(-j \boldsymbol{\omega}^{\mathrm{T}} \mathbf{x}\right) \mathrm{d} \mathbf{x}$. We also define the discrete autocorrelation sequence $a_{f}$ of $f$ by $a_{f}[\mathbf{k}]=(\bar{f} * f)(\mathbf{R k})$, where $\bar{f}(\mathbf{x})=f(-\mathbf{x})$. Convolutions are denoted by $*$, and a star * indicates the complex conjugate. $f(\mathbf{x})=O(g(\mathbf{x}))$ means that $\limsup _{\|\mathbf{x}\| \rightarrow 0}|f(\mathbf{x}) / g(\mathbf{x})|<\infty$ and $f(\mathbf{x}) \sim g(\mathbf{x})$ denotes the equivalence $\lim _{\|\mathbf{x}\| \rightarrow 0} f(\mathbf{x}) / g(\mathbf{x})=1$.

The effect of sampling a function $f(\mathbf{x})$ on the hexagonal lattice is to replicate its spectrum $\hat{f}(\boldsymbol{\omega})$ at the lattice sites $2 \pi \widehat{\mathbf{R}} \mathbf{k}$, where $\widehat{\mathbf{R}}=\left(\mathbf{R}^{-1}\right)^{\mathrm{T}}$. Accordingly, the Fourier transform of a discrete signal $s=(s[\mathbf{k}])_{\mathbf{k} \in \mathbb{Z}^{2}}$ sampled on the hexagonal lattice is $\hat{s}(\boldsymbol{\omega})=\sum_{\mathbf{k} \in \mathbb{Z}^{2}} s[\mathbf{k}] \exp \left(-j \boldsymbol{\omega}^{\mathrm{T}} \mathbf{R} \mathbf{k}\right)$. With $s^{-1}$, we denote the signal with Fourier transform $1 / \hat{s}(\boldsymbol{\omega})$. The $\mathcal{Z}$-transform of $s$ is $S(\mathbf{z})=\sum_{\mathbf{k} \in \mathbb{Z}^{2}} s[\mathbf{k}] \mathbf{z}^{-\mathbf{k}}$, where $\mathbf{z}^{-\mathbf{k}}=z_{1}^{-k_{1}} z_{2}^{-k_{2}}$. For a more complete overview on lattices and signals defined on them, we refer to $[2,7]$.

Interpolating the discrete signal $s$ using a generator $\varphi(\mathbf{x})$ consists in reconstructing the function (see [6])

$$
f_{\text {int }}(\mathbf{x})=\sum_{\mathbf{k} \in \mathbb{Z}^{2}} c[\mathbf{k}] \varphi(\mathbf{x}-\mathbf{R} \mathbf{k}),
$$

where the coefficients $c[\mathbf{k}]$ are obtained by prefiltering the data, in order to satisfy the interpolation condition $f_{\text {int }}(\mathbf{R} \mathbf{k})=s[\mathbf{k}]$ for every $\mathbf{k} \in \mathbb{Z}^{2}$; That is, $c=s * p_{\text {int }}$ using the interpolation prefilter 


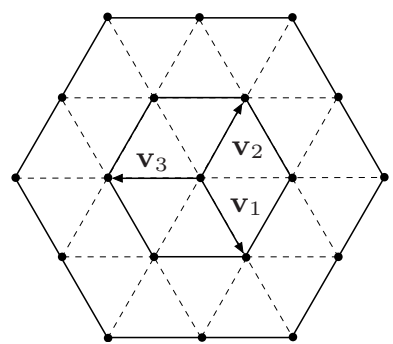

(a)

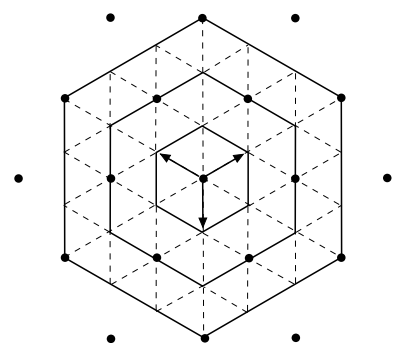

(b)
Fig. 1. The box-splines $\chi_{2 n}$ (a) and hex-splines $\eta_{L}$ (b) are piecewise polynomial over triangles (dotted lines). Their supports are hexagons (solid lines) with surface area $3 n^{2}$ and $L^{2}$, respectively.

defined by $p_{\text {int }}^{-1}[\mathbf{k}]=\varphi(\mathbf{R k})$. A Fourier domain implementation is appropriate for this prefiltering step, see e.g. [8, Appendix E]. Note that in practical applications, most of the computation time is consumed not by the prefiltering step, but by the reconstruction (2) itself. In fact, the size of the support of $\varphi$ is the most crucial element that determines the computational complexity of an interpolation algorithm [6].

The three directional box-splines and hex-splines are piecewise polynomial functions that form two different multi-dimensional extensions of the 1-D B-splines, appropriate for interpolation on the hexagonal lattice. Among the vast family of box-splines, the threedirectional ones are non-separable and twelve-fold symmetric, hence adapted to the hexagonal lattice. They can be defined by $\widehat{\chi}_{2 n}(\boldsymbol{\omega})=$ $\prod_{i=1}^{3} \operatorname{sinc}\left(\omega^{\mathrm{T}} \mathbf{v}_{i} / 2\right)^{n}$, where $n \geq 1, \operatorname{sinc}(x)=\sin (x) / x$ and

$$
\mathbf{v}_{1}=\sqrt{\frac{2}{\sqrt{3}}}\left[\begin{array}{c}
1 / 2 \\
-\sqrt{3} / 2
\end{array}\right], \mathbf{v}_{2}=\sqrt{\frac{2}{\sqrt{3}}}\left[\begin{array}{c}
1 / 2 \\
\sqrt{3} / 2
\end{array}\right], \mathbf{v}_{3}=\sqrt{\frac{2}{\sqrt{3}}}\left[\begin{array}{c}
-1 \\
0
\end{array}\right],
$$

as depicted in Fig. 1. Recently, the authors derived an explicit analytical formula for $\chi_{2 n}$ with arbitrary $n$, along with an efficient implementation [9].

Hex-splines are another family of functions, proposed recently in [8], built by successive convolution: $\eta_{L}=\eta_{L-1} * \eta_{1}$, for every $L>1$, where the first-order hex-spline $\eta_{1}$ is simply the indicator function of the Voronoi cell of the lattice $\Lambda$. The expressions of the hex-splines in the Fourier domain, as well as their properties, are given in [8] and [10]. $\eta_{L}(\mathbf{x})$ has a compact hexagonal support, as shown in Fig. 1.

Box-splines [11] have numerous practical applications, e.g. in geometric modelling and multiscale representation systems [12] while hex-splines found applications in printing [13]. For a plot of the first orders box- and hex-splines, see [10, Fig. 3]. Note that due to the convolution properties, the discrete autocorrelations satisfy $a_{\eta_{L}}[\mathbf{k}]=\eta_{2 L}(\mathbf{R k})$ and $a_{\chi_{2 n}}[\mathbf{k}]=\chi_{4 n}(\mathbf{R k})$.

\section{NEW HEXAGONAL SPLINE GENERATORS}

\subsection{Assessment of the Interpolation Error}

Our goal is to construct new functions $\varphi$ showing an interpolation error $e^{2}=\int_{\mathbb{R}^{2}}\left|f(\mathbf{x})-f_{\text {int }}(\mathbf{x})\right|^{2} \mathrm{~d} \mathbf{x}$ smaller than with hex-splines and box-splines. A remarkable result of approximation theory tells us that this error can be estimated very accurately by means of the error kernel $E(\boldsymbol{\omega})$ in the frequency domain (see [14] for the exact meaning of " $\approx$ " in $(4)$; exact equality holds in many situations, as well as in a stochastic sense):

$$
e^{2} \approx \frac{1}{(2 \pi)^{2}} \int_{\mathbb{R}^{2}}|\hat{f}(\boldsymbol{\omega})|^{2} E(\boldsymbol{\omega}) \mathrm{d} \boldsymbol{\omega},
$$

where

$$
E(\boldsymbol{\omega})=1-\frac{|\hat{\varphi}(\boldsymbol{\omega})|^{2}}{\hat{a}_{\varphi}(\boldsymbol{\omega})}+\hat{a}_{\varphi}(\boldsymbol{\omega})\left|\hat{p}_{\mathrm{int}}(\boldsymbol{\omega})-\frac{\hat{\varphi}(\boldsymbol{\omega})^{*}}{\hat{a}_{\varphi}(\boldsymbol{\omega})}\right|^{2} .
$$

In practice, most energy of images is concentrated in the lowfrequency part of the spectrum, which implies that the error is dominated by the behavior of the error kernel at the origin. An asymptotic analysis at $\boldsymbol{\omega}=\mathbf{0}$ yields, in polar coordinates $\boldsymbol{\omega}=$ $(\|\boldsymbol{\omega}\| \cos (\theta),\|\boldsymbol{\omega}\| \sin (\theta))$,

$$
E(\boldsymbol{\omega}) \sim C_{\text {int }}(\theta)\|\boldsymbol{\omega}\|^{2 L},
$$

where $C_{\text {int }}$ is called the asymptotic constant of $\varphi[14]$ and $L$ is the approximation order of $\varphi$, defined by the Strang-Fix conditions [15]:

$$
\hat{\varphi}(\mathbf{0}) \neq 0 \text { and } \hat{\varphi}(\boldsymbol{\omega}-2 \pi \widehat{\mathbf{R}} \mathbf{k})=O\left(\|\boldsymbol{\omega}\|^{L}\right) \text { for every } \mathbf{k} \neq \mathbf{0} .
$$

Note that the subscript numbers of $\chi_{2 n}$ and $\eta_{L}$ are their respective approximation orders.

Thus, it is desirable to choose $\varphi$ having an approximation order as high as possible, since this parameter determines the flatness of the error kernel around the origin, hence the ability to reproduce the low-frequency content of $f$ with small error. Therefore, the approximation order is the main parameter that rules the interpolation quality, as shown by many numerical evidences $[6,16,10]$. However, the size of the support of $\varphi$ grows with $L$, thus a tradeoff between interpolation quality and computational complexity has to be achieved.

\subsection{Construction of the new families of functions}

In the 1-D case, it has been showed that there is a closed family of functions having the shortest support, of size $L$, for a given approximation order $L$ [1]. These functions are called "MOMS", for "maximum order with minimal support". They are optimal, since they achieve the best compromise between interpolation quality and computational complexity $[1,6]$. The centered B-spline of degree $n$, $\beta^{n}(x)$, is a MOMS. In fact, every MOMS with approximation order $L$ can be expressed as a linear combination of $\beta^{L-1}(x)$ and its successive derivatives. That is, a normalized symmetrical MOMS $\varphi(x)$ with approximation order $L$ satisfies

$$
\hat{\varphi}(\omega)=\hat{\beta}^{L-1}(\omega)+\sum_{i=1}^{(L-1) / 2} a_{i} \hat{h}(\omega)^{i} \hat{\beta}^{L-2 i-1}(\omega)
$$

for some coefficients $a_{i}$, where $h$ is the second-order finite difference filter $[-12-1]$ (that is, $\hat{h}(\omega)=2-2 \cos (\omega)$ ).

Inspired by the 1-D construction of MOMS, we propose new functions, that we note $B M_{L}(\mathbf{x})$ and $H M_{L}(\mathbf{x})$, that extend the families of box-splines and hex-splines, respectively. In order to obtain a construction similar to the form (8), we first assess the two following properties.

PROPERTY 1: Let us consider two 2-D functions $\phi_{1}$ and $\phi_{2}$ both with convex compact support, and a filter $h$ such that $h[\mathbf{k}] \neq 0$ only at the sites Rk included in the support of $\phi_{1}$ (or on its boundary). We define $\phi_{3}=\phi_{1} * \phi_{2}$ and $\phi_{4}=\sum_{\mathbf{k} \in \mathbb{Z}^{2}} h[\mathbf{k}] \phi_{2}(\mathbf{x}-\mathbf{R} \mathbf{k})$. Then, the support of $\phi_{4}$ is included in the one of $\phi_{3}$. 
Proof: Let $\mathbf{x}_{0}$ be such that $\phi_{3}\left(\mathbf{x}_{0}\right)=0$. Since $\phi_{3}\left(\mathbf{x}_{0}\right)=\int_{\mathbb{R}^{2}} \phi_{1}(\mathbf{x}) \phi_{2}\left(\mathbf{x}_{0}-\mathbf{x}\right) \mathrm{d} \mathbf{x}$ then the supports of $\phi_{1}$ and $\phi_{2}\left(\mathbf{x}_{0}-\cdot\right)$ are disjoint. Consequently, if $\phi_{1}(\mathbf{R k}) \neq 0$, then $\phi_{2}\left(\mathbf{x}_{0}-\mathbf{R} \mathbf{k}\right)=0$. Hence, $\phi_{4}\left(\mathbf{x}_{0}\right)=0$.

PROPERTY 2: Let us consider two functions $\phi_{1}$ and $\phi_{2}$, with approximation order $L_{1}$ and $L_{2} \leq L_{1}$ respectively, and a filter $h$ (localized on $\Lambda$ ) such that $\hat{h}(\mathbf{0})=O\left(\|\boldsymbol{\omega}\|^{L_{1}-L_{2}}\right)$. Then $\phi_{3}=$ $\phi_{1}+\sum_{\mathbf{k} \in \mathbb{Z}^{2}} h[\mathbf{k}] \phi_{2}(\mathbf{x}-\mathbf{R k})$ has approximation order $L_{1}$.

Proof: $\widehat{\phi_{3}}(\boldsymbol{\omega})=\widehat{\phi_{1}}(\boldsymbol{\omega})+\hat{h}(\boldsymbol{\omega}) \widehat{\phi_{2}}(\boldsymbol{\omega})$. Since $\hat{h}(\boldsymbol{\omega})$ is $2 \pi \hat{\Lambda}$-periodic, we can check that the Strang-Fix conditions of order $L_{1}$ in (7) are satisfied for $\widehat{\phi_{3}}$.

We now introduce the fiter $h$ depicted in Fig. 2 (c). Equivalently, $H(\mathbf{z})=6-\left(z_{1}+z_{2}+z_{2} z_{1}^{-1}+z_{1}^{-1}+z_{2}^{-1}+z_{1} z_{2}^{-1}\right)$. For every $L \geq 1$, we define a box-moms $B M_{L}(\mathbf{x})$ (if $L$ is even) and a hexmoms $H M_{L}(\mathbf{x})$ as a function having the respective form

$$
\begin{aligned}
& \widehat{B M}_{L}(\boldsymbol{\omega})=\widehat{\chi}_{L}(\boldsymbol{\omega})+\sum_{i=1}^{(L-1) / 2} a_{i} \hat{h}(\boldsymbol{\omega})^{i} \widehat{\chi}_{L-2 i}(\boldsymbol{\omega}), \\
& \widehat{H M}_{L}(\boldsymbol{\omega})=\widehat{\eta}_{L}(\boldsymbol{\omega})+\sum_{i=1}^{(L-1) / 2} a_{i} \hat{h}(\boldsymbol{\omega})^{i} \widehat{\eta}_{L-2 i}(\boldsymbol{\omega}),
\end{aligned}
$$

for some coefficients $a_{i}$. One can easily check that $\hat{h}(\boldsymbol{\omega})=$ $O\left(\|\boldsymbol{\omega}\|^{2}\right)$. Moreover, $h$ has its support included in the ones of $\chi_{2}(\mathbf{x})$ and $\eta_{2}(\mathbf{x})$. As a consequence of the Properties 1 and 2, $B M_{L}(\mathbf{x})$ and $H M_{L}(\mathbf{x})$ have approximation order $L$ and the same support as $\chi_{L}(\mathbf{x})$ and $\eta_{L}(\mathbf{x})$, respectively, see Fig. 1. However, neither the splines nor the new functions are true MOMS, in the sense that holds in 1-D. It can be conjectured that a 2-D function of approximation order $L$ should have a support of minimal size $L(L+1) / 2$, but this is an open question, to our knowledge.

\subsection{Approximation-theoretically optimal functions}

We now focus on the issue of choosing, among the hex-moms or box-moms family of given order $L$, an "optimal" representant; that is, we look for the best coefficients $a_{i}$ in (9) or (10). Our purpose is to minimize the error kernel $E(\boldsymbol{\omega})$ associated to $\varphi$, that rules the interpolation quality; hence, we want to minimize the asymptotic constant $C_{\text {int }}$ in (6). Actually, numerical evidences have shown that the minimization of the asymptotic constant, among a family of functions having the same approximation order $L$, yields significant improvements for image processing tasks $[1,10]$.

For an approximation order $L \leq 2$, there is no free parameter in (10) and (9), and the hex-moms and box-moms revert to hex-splines and box-splines. Since, in practical applications, $L=4$ may provide a sufficient quality, while the implementation may become burdensome for higher values of $L$, we concentrate on designing an optimal hex-moms $\mathrm{HM}_{3}(\mathbf{x})$ and an optimal box-spline $H M_{4}(\mathbf{x})$. We can write them in the spatial domain as

$$
H M_{3}=\eta_{3}(\mathbf{x})+\alpha \sum_{\mathbf{k} \in \mathbb{Z}^{2}} h[\mathbf{k}] \eta_{1}(\mathbf{x}-\mathbf{R} \mathbf{k})
$$

with associated constant, obtained after some calculations using Taylor series in (5):

$$
C_{\mathrm{int}}(\theta)=\frac{5 \sqrt{3}}{36}\left(\alpha+\frac{7}{1800}\right)^{2}+\frac{7039-1595 \cos (6 \theta)}{4408992000} \sqrt{3}
$$

\begin{tabular}{l|ccccccc}
\hline$\varphi$ & $\eta_{1}$ & $\chi_{2}$ & $\eta_{2}$ & $\eta_{3}$ & $\chi_{4}$ & $H M_{3}$ & $B M_{4}$ \\
\hline Lena & 35.86 & 42.16 & 41.87 & 46.75 & 47.16 & 46.78 & 47.63 \\
Barbara & 29.24 & 33.60 & 33.31 & 39.64 & 40.77 & 39.85 & 41.85 \\
Baboon & 28.09 & 31.88 & 31.57 & 36.31 & 36.88 & 36.38 & 37.60 \\
Lighthouse & 29.50 & 34.92 & 34.65 & 41.44 & 42.43 & 41.60 & 43.55 \\
Goldhill & 34.61 & 39.39 & 39.10 & 44.15 & 44.74 & 44.22 & 45.44 \\
Boat & 32.82 & 37.75 & 37.48 & 41.63 & 41.91 & 41.63 & 42.28 \\
Peppers & 35.33 & 39.66 & 39.12 & 42.95 & 43.09 & 42.89 & 43.35 \\
\hline time, in $s$ & 0.1 & 0.1 & 0.3 & 1.4 & 1.4 & 1.4 & 1.4 \\
\hline
\end{tabular}

Table 1. PSNR obtained for the resampling experiments between the Cartesian and hexagonal lattices, as described in Section 4.

and

$$
B M_{4}=\chi_{4}(\mathbf{x})+\beta \sum_{\mathbf{k} \in \mathbb{Z}^{2}} h[\mathbf{k}] \chi_{2}(\mathbf{x}-\mathbf{R k})
$$

with associated constant

$C_{\text {int }}(\theta)=\frac{193}{3135283200}+\frac{3}{160}\left(\beta+\frac{11}{1296}\right)^{2}-\frac{\cos (6 \theta)}{7257600}-\frac{\beta \cos (6 \theta)}{60480}$.

Therefore, the desired value providing the optimal hex-moms of order 3 is $\alpha=-7 / 1800$. For the box-moms, we choose to minimize the average value of $C_{\text {int }}(\theta)$ over $\theta$. This amounts to minimizing the asymptotic interpolation error for isotropic signals. Hence, our optimal box-moms of order 4 is defined with $\beta=-11 / 1296$. An alternative design would be to look for $\beta$ such that $C_{\text {int }}(\theta)$ is independant of $\theta$, to enforce an asymptotic interpolation quality equal in every direction. This yields $\beta=-1 / 120$, which is very close to our adopted value for this parameter.

We have to mention that, in another work [10], we used the same minimization process to optimize, for a given generator, the prefiltering step $c=s * p$ yielding the coefficients $c[\mathbf{k}]$ in (2); that is, the interpolation prefilter $p_{\text {int }}$ was replaced by a quasi-interpolating prefilter. The reconstructed function does not interpolate the samples $s[\mathbf{k}]$ any more, but the reconstruction error is reduced. This approach may be combined with the one in the present work, for global optimization of both the function $\varphi$ and the prefilter $p$, in situations where it is not necessary to enforce the exact interpolation condition. Also, we can find optimal "moms" functions for other reconstruction situations, like orthogonal or oblique projections in the reconstruction space, instead of interpolation.

\section{EXPERIMENTAL VALIDATION}

In order to quantify numerically the gain offered by our new functions, we propose the following resampling setting. First, a standard test image is resampled from its supporting Cartesian lattice to the hexagonal one with same density, using separable cubic O-MOMS interpolation, whose high fidelity is well known $[1,6]$. The samples $s[\mathbf{k}]$ of this new image, located on the hexagonal lattice, are considered as input for the proposed interpolation methods. The interpolated function $f_{\text {int }}(\mathbf{x})$ is then resampled on the initial Cartesian lattice, defining a final image that is compared to the reference image. We applied this setting to seven classical $512 \times 512$ images of the literature, using mirror conditions at the boundaries. The PSNR measures between the resampled and ground-truth images for this test set are summarized in Tab. 1. The last row of this table indicates computation times, for a $\mathrm{C}$ code running on a $1.6 \mathrm{GHz} \mathrm{PC}$ ). 


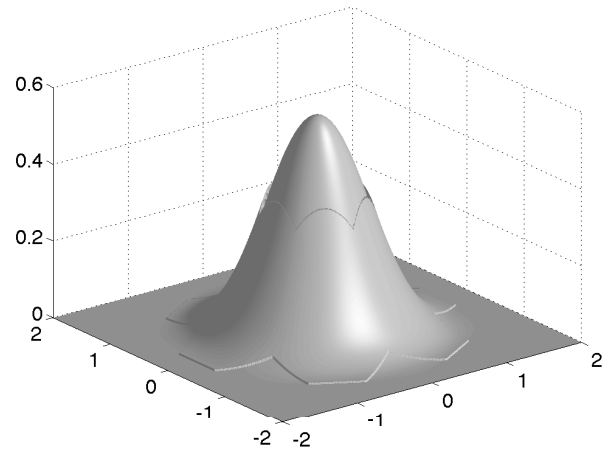

(a)

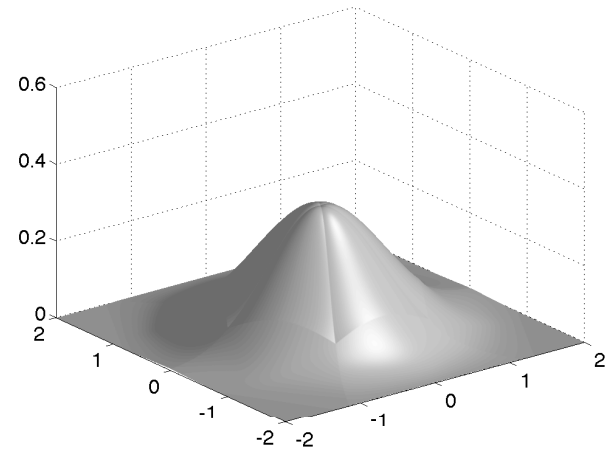

(b)

$$
\begin{aligned}
& \begin{array}{ll}
-1 & -1
\end{array} \\
& h=-1 \quad 6 \quad-1 \\
& \begin{array}{ll}
-1 & -1
\end{array}
\end{aligned}
$$

(c)

Fig. 2. (a) hex-moms function $H M_{3}(\mathbf{x})$ and (b) box-moms function $B M_{4}(\mathbf{x})$, both with optimal parameters $\alpha$ and $\beta$ determined in Sect. 3.3. In (c), the finite difference filter used for their construction.

We considered for generators in our test the hex-splines of order 1 to 3, the box-splines of order 2 and 4, and our two new generators $\mathrm{HM}_{3}(\mathbf{x})$ and $B M_{4}(\mathbf{x})$. As a result, the hierarchy between the generators with respect to the approximation order is respected, as predicted by the theory. The gain provided by $H M_{3}$ over $\eta_{3}$ is small $\left(0.07 \mathrm{~dB}\right.$ in average), but the one of $B M_{4}$ over $\chi_{4}$ is quite substantial $\left(0.69 \mathrm{~dB}\right.$ in average). Interpolation with $B M_{4}$ also outperforms the combination of $\chi_{4}$ with the best quasi-interpolation prefilter proposed in [10].

We insist on the fact that the quality improvement of our approach is obtained at no expense, since the new functions and their spline counterparts have the same supports and polynomial degrees; hence, the computation cost is exactly the same.

\section{CONCLUSION}

We proposed in this work a generic method for designing new generators, well suited for interpolation on the hexagonal lattice, by enlarging existing families of multi-dimensional splines. By minimizing the asymptotic approximation error, we obtained new functions with superior performances over their spline counterparts, for the same computation time. The ideas are straightforward to extend to any lattice and in any dimension; for instance, a box-moms extension of the 3-D box-spline deployed on the BCC lattice could yield great benefits for high-quality visualization of 3-D data [17].

\section{REFERENCES}

[1] T. Blu, P. Thévenaz, and M. Unser, "MOMS: Maximal-order interpolation of minimal support," IEEE Trans. Image Processing, vol. 10, no. 7, pp. 1069-1080, July 2001.

[2] R. M. Mersereau, "The processing of hexagonally sampled two-dimensional signals," Proc. IEEE, vol. 67, no. 6, pp. 930949, June 1979.

[3] L. Condat, D. Van De Ville, and T. Blu, "Hexagonal versus orthogonal lattices: A new comparison using approximation theory," in Proc. of IEEE ICIP, vol. 3, Sept. 2005.

[4] S. Jung, R. Thewes, T. Scheiter, K. F. Goser, and W.Weber, "Low-power and high-performance CMOS fingerprint sensing and encoding architecture," IEEE J. Solid-State Circuits, vol. 34, pp. 978-984, July 1999.
[5] M. Unser, "Splines: A perfect fit for signal and image processing," IEEE Signal Processing Mag., vol. 16, no. 6, pp. 22-38, Nov. 1999.

[6] P. Thévenaz, T. Blu, and M. Unser, "Interpolation revisited," IEEE Trans. Med. Imag., vol. 19, no. 7, pp. 739-758, July 2000.

[7] D. E. Dudgeon and M. E. Mersereau, Multidimensional Signal Processing. Englewood Cliffs, NJ: Prentice-Hall, 1984.

[8] D. Van De Ville, T. Blu, M. Unser, W. Philips, I. Lemahieu, and R. Van De Walle, "Hex-spline: A novel family for hexagonal lattices," IEEE Trans. Image Processing, vol. 13, no. 6, pp. 758-772, June 2004.

[9] L. Condat and D. Van De Ville, "Three-directional box-splines: Characterization and efficient evaluation," IEEE Signal Processing Lett., vol. 13, no. 7, pp. 417-420, July 2006.

[10] — - "Quasi-interpolating spline models for hexagonallysampled data," IEEE Trans. Image Processing, vol. 16, no. 5, pp. 1195-1206, May 2007.

[11] C. de Boor, K. Höllig, and S. Riemenschneider, Box Splines. Berlin: Springer-Verlag, 1993, vol. Applied Mathematical Sciences, vol. 98.

[12] H. Prautzsch and W. Boehm, "Box splines," in Handbook of Computer Aided Geometric Design. Berlin: Springer, 2001.

[13] D. Van De Ville, W. Philips, and I. Lemahieu, "Least-squares spline resampling to a hexagonal lattice," Signal Processing: Image Communication, vol. 17, no. 5, pp. 393-408, May 2002.

[14] T. Blu and M. Unser, "Quantitative Fourier analysis of approximation techniques: Part I-interpolators and projectors and Part II-wavelets," IEEE Trans. Signal Processing, vol. 47, no. 10, pp. 2783-2806, Oct. 1999.

[15] G. Strang and G. Fix, "A Fourier analysis of the finite element variational method," in Constructive aspect of functional analysis, Rome, Italy: Cremonese, 1971, pp. 796-830.

[16] E. Meijering, W. Niessen, J. Pluim, and M. Viergever, "Quantitative comparison of sinc-approximating kernels for medical image interpolation," in Proc. of MICCAI, 1999, pp. 210-217.

[17] A. Entezari, R. Dyer, and T. Möller, "Linear and cubic box splines for the body centered cubic lattice," in Proc. of IEEE Visualization, Austin, TX, Oct. 2004, pp. 11-18. 